\title{
Reliability and validity of the pediatric feeding and swallowing disorders family impact scale for Turkish children with cerebral palsy by endoscopic evaluation
}

\author{
Ebru Umay, İbrahim Gündoğdu, Erhan Arif Öztürk \\ University of Health Sciences, Ankara Diskapi Yildirim Beyazit Education and Research Hospital, Physical Medicine and \\ Rehabilitation Clinic, Ankara, Turkey.E-mail: ebruumay@gmail.com \\ Received: 13th November 2018, Accepted: 23rd January 2019
}

SUMMARY: Umay E, Gündoğdu İ, Öztürk EA. Reliability and validity of the pediatric feeding and swallowing disorders family impact scale for Turkish children with cerebral palsy by endoscopic evaluation. Turk J Pediatr 2019; 61: 741-748.

The caregivers of children with cerebral palsy (CP) have high mood disorders and stress levels. This study was aimed to conduct validity and reliability of Turkish version of The Pediatric Feeding and Swallowing Disorders Family Impact Scale (PFSDFIS) by using an objective method. This study was performed in our physical medicine and rehabilitation (PMR) clinic between July 2016 and July 2018. This study was performed with 251 children with $\mathrm{CP}$ who had complaint of swallowing and/or feeding problems, and their primer caregivers. Cronbach's alpha and corrected item-total correlations were used to assess internal consistency. Test and retest reliability studies were also conducted. The construct validity was assessed using the dysphagia level defined with flexible fiberoptic endoscopic evaluation of swallowing and Impact on Family Scale (IFS). Total score of T-PFSDFIS was correlated to the dysphagia level by using FEES. Results showed, Cronbach's alpha value of the scale to be 0.821 . Corrected item-to-total correlations ranged from 0.729 to 0.911 . Test-retest reliability coefficients was calculated with intra-class correlation coefficient (ICC), the total score was 0.989. A negative significant good level correlation was found between the dysphagia level by using endoscopic evaluation and the T- PFSDFIS total score as well as between total scores of IFS and T-PFSDFIS. In subgroup analysis; the lowest value was in normal swallowing and significantly different from all dysphagia levels. In conclusion; this scale is effective in reflecting the influence of caregivers on the severity of dysphagia measured objectively and T-PFSDFIS is a valid and reliable scale for Turkish children with $\mathrm{CP}$.

Key words: cerebral palsy, caregiver, the Pediatric Feeding and Swallowing Disorders Family Impact Scale, reliability, validity.

Cerebral palsy (CP) is a permanent, nonprogressive disease characterized by disorders of movement and posture. Recent advances in neonatal care have resulted in a significant improvement in the survival of premature infants but enhanced survival has been accompanied by a significant increase in the incidence of CP. ${ }^{1}$ The incidence of swallowing disorders in children is also reported to be increasing secondary to improved survival rates and the improved life expectancy of children with CP. ${ }^{1}$ The motor disorders of $\mathrm{CP}$ are often accompanied by disturbances of sensation, cognition, perception communication or behavior or secondary musculoskeletal problems. ${ }^{2}$ Swallowing disorders can be seen in these children at varying degrees from mild to severe. In has been reported in the literature that the most important factors determining life expectancy in CP are lack of mobility and feeding. ${ }^{3}$

Dysphagia has many consequences for both 
children and their caregivers. ${ }^{4,5}$ Dysphagia in children may result in several complications, including dehydration, malnutrition, airway obstruction, aspiration pneumonia, and death. ${ }^{6}$ Therefore, many studies have been done to understand the physiology of swallowing in children. Examination methods and instrumental methods were used for this purpose.

Despite these developments in understanding the anatomy and physiology of swallowing disorders, there is little research investigating the effects of these problems on the caregiver and the child's daily life. In addition to medical problems, eating disorders may lead to higher levels of perceived stress that affect the psychosocial lives of the child, family and people around it. ${ }^{7}$ It is useful, therefore, for clinicians to understand the impact of these problems and to improve the formulation of solutions helping the family and the child.

The prevalence of $\mathrm{CP}$ was reported as 4.4 per 1000 live births in Turkey. ${ }^{8,9}$ Studies also have shown that dysphagia is common in these children. ${ }^{10,11}$ Moreover, there are studies showing that there are mood disorders and stress levels such as severe anxiety and depression in the caregivers of these children with dysphagia. ${ }^{11}$ Although there are caregiver impact scales adapted to Turkish, there is currently no valid and reliable tool especially measuring caregiver impact for $\mathrm{CP}$ children with dysphagia in Turkey.

Hence, the aim of this study was to conduct the validity and reliability study of the Turkish version of The Pediatric Feeding and Swallowing Disorders Family Impact Scale (T-PFSDFIS); an instrument designed to measure and improve the impact feeding and swallowing disorders have on caregivers .

\section{Material and Methods}

\section{Study Design and Patients}

This study was performed with 257 children with CP who had complaint of swallowing and/or feeding problems, and their primer caregivers who were followed-up in our physical medicine and rehabilitation (PMR) clinic between July 2016 and July 2018.
Six caregivers who did not participate in the re-test phase of study were excluded. Thus, the study was completed with 251 children with CP and their caregivers.

The study was approved by the local Institutional Ethics Committee. The caregivers were given verbal and written information of the before study. Informed consent forms were signed upon admission to the trial. All procedures were conducted in accordance with the Helsinki Declarations of 2004.

The inclusion criteria were children diagnosed with CP between the ages of 4 and 8 years of age and the exclusion were any patient who had received head and neck surgery, received a Botulinum toxin $\mathrm{A}$ injection in the previous 6 months and had an infection that could affect swallowing during examination. Also, the children who had the presence of contagious or infectious diseases, nasal obstruction, decompensated heart disease, and any risk of bleeding were excluded due to contraindications for flexible fiberoptic endoscopic evaluation of swallowing (FEES).

The inclusion criteria for caregivers were; caregivers who were the child's primer caregiver, who had sufficient cognitive ability to understand questions, who were functionally independent and who had no problem that could affect their ability to care, as well as those who agreed to participate in the study.

The children and their caregivers who did not meet the inclusion criteria were excluded from the study.

\section{Data Collection}

\section{Sample size}

The sample size calculation was derived from power analysis and sample size software. The required sample size was 178 children with CP for the reliability and validity of the The PFSDFIS (medium effect size $(d=0.5)$, 0.05 type I error rate and $80 \%$ significantly power value). Initially, sampling adequacy was assessed using the Kaiser-Meyer-Olkin (KMO) test and the Bartlett's test of sphericity. The KMO test and Bartlett's test of sphericity showed that the data were adequate for 
reliability and validity analysis $\left(0.988\right.$ and $\mathrm{X}^{2}$ $=2138.11, \mathrm{df}=204, \mathrm{p}<0.001$, respectively).

\section{Caregiver demographics}

Demographic information for caregivers included age, gender, education and marital status. Additional family information included the total number of people and total number of children in the house where child lives and family income.

\section{Child demographics and disease characteristics}

Demographic characteristics including age, gender, height, weight, body mass index (BMI), additional problems such as mental delay, seizure, hearing, vision, speech, gastrointestinal and dental disorders, and current feeding method (oral and enteral such as nasogastric or percutaneous gastrostomy tubes) were recorded. Also, involvement types of CP were identified as hemi-, di- and tetraparesis and motor development was graded using the Gross Motor Function Measure (GMFM) as between I and V.

\section{Instrument}

\section{The Pediatric Feeding and Swallowing Disorders Family Impact Scale (PFSDFIS)}

The Pediatric Feeding and Swallowing Disorders Family Impact Scale (PFSDFIS) was developed to help clinicians identify the impact feeding and swallowing problems have on the family by Redle. ${ }^{12}$ The tool addresses specific concerns and needs of these families. Clinical researchers could also use this scale for outcomes measurement. During the development phase, 50 questions were created and then 44 questions were revised. This scale includes five subscales; general impact on the family and child, mealtime satisfaction, social impact and community interactions. The 5 -points Likert type scale was used between "strongly agree (1)" and "strongly disagree (5)". The revised-scale was found to be reliable and valid in 90 children with chronic illnesses but translation, validity and reliability studies have not been conducted in other languages. The total score ranges from 44 to 220 , low scores indicating greatest effect on family.

\section{Translation Procedures}

Translation procedures were performed according to the report of Beaton et al. ${ }^{13}$ Permission to use and translate the questionnaire was obtained from the author Redle et al. ${ }^{12}$ The PFSDFIS was independently translated into Turkish by 4 PMR specialists. After comparing all translations and making necessary corrections, one consensus text was created. It was then translated into English in collaboration with 2 professional linguists. The final Turkish-PFSDFIS (T- PFSDFIS) was accepted following a comparison of the meaning and format with the original English form. After the pilot study was completed on target population (10 patients and their caregivers), the form was finalized by the feedback obtained.

\section{Reliability}

Cronbach's alpha and corrected item-total correlations were used to assess internal consistency. Test and retest reliability studies were also conducted. The test-retest reliability was calculated on the answers of 251 caregivers who were able to complete the questionnaire twice with an interval of one week by using intra-class correlation coefficient (ICC).

\section{Validity}

The construct validity was assessed using the dysphagia level defined with flexible fiberoptic endoscopic evaluation of swallowing (FEES) and Impact on Family Scale (IFS). Also, total score of T-PFSDFIS was correlated to the dysphagia level by using FEES.

\subsubsection{Endoscopic evaluation (FEES)}

Endoscopic evaluation was performed by an otolaryngology specialist blinded to the design of the trial within the first 24 hours after performing the retest. Endoscopic evaluation was performed using a non-ducted fiberoptic nasopharyngoscope of $3.4 \mathrm{~mm}$ diameter, a light source, camera and monitor. (Karl Storz $\mathrm{GmbH} \&$ Co KG, Tuttlingen, Germany). The assessments were performed with the patient at the highest possible upright sitting position. Water was used as the liquid nutriment, liquid with food thickener as the semisolid and bread as the solid food during evaluations. Findings 
were recorded as video images. Dysphagia level was scored from 1 to 6 according to the protocol of assessment of dysphagia developed by Warnecke et al. ${ }^{14}$ In which 1 point is considered as "normal swallowing", 2-3 points as "mild", 4-5 points as "moderate", and 6 points as "severe" dysphagia.

\section{Impact on Family Scale (IFS)}

The Impact on Family Scale (IFS) was initially developed to quantify the impact of pediatric chronic illnesses on parents and families. ${ }^{15}$ The Impact on Family Scale is a 27 -item inventory and all items have a standard four-point scale ranging from strongly agree to strongly disagree similar to PFSDFIS. Higher scores indicate greatest impact on the family.

\section{Statistical Analysis}

All statistical analyses were carried out by using SPSS 20.0 for windows statistical package. The missing data (\%) and the computable points (\%) were determined for the data quality. The incomplete data rate $<5 \%$ was determined as the acceptable level. Descriptive statistics were demonstrated as mean (standard deviation (SD)) for continuous variables and as a percentage (\%) for nominal variables. Internal consistency was measured using Cronbach's alpha, $>0.70$ indicating an acceptable value. Corrected item-total correlations were calculated by using Spearman rho correlation coefficients. Correlation coefficients $\geq 0.3$ were considered as acceptable. ${ }^{16}$ Test and retest reliability was estimated using ICC. According to ICC results, positive values ranging from 0 to 0.2 indicate poor agreement; 0.2 to 0.4 indicate fair agreement; 0.4 to 0.6 indicate moderate agreement; 0.6 to 0.8 indicate good agreement; and 0.8 to 1 indicate very good agreement. ${ }^{17}$ Floor and ceiling effects were determined for acceptability. The effect of the floors or ceilings $<15 \%$ was defined as acceptable. For validity, Spearman rho correlation test was used to indicate an association between FEES and IFS, and total score of T-PFSDFIS. The correlation coefficient ( $r$ ) was used to show the power of correlation. According to this; $<0.30$ points indicated weak, 0.30 to 0.50 points indicated moderate, 0.50 to 0.75 points indicated good correlation, 0.75 to 1.0 point indicated a very good correlation between the variables. Subgroup analysis for FEES level was performed ANOVA test. $\mathrm{P}<0.05$ values were accepted as statistically significant.

\section{Results}

\section{Children and Caregiver Characteristics}

The mean age of caregivers was 34.24 years (SD 10.55), 249 (99.2\%) were female and $2(0.8 \%)$ were male. The mean age of 251 children was 68.11 (SD 20.26) months, 79 (31.5\%) were girls and $172(68.5 \%)$ were boys. Also, the mean GMFM level was 4.15 (SD 0.43). The mean dysphagia level of children detected by FEES was 3.75 (SD 1.18). Twenty-nine $(11.6 \%)$ of children were normal with FEES. The caregivers' and children characteristics are shown in Table I and Table II.

\section{Summary of T-PFSDFIS}

There was no missing data in any item of the scale and all of the data was used for statistical analysis.

The total T- PFSDFIS score was 122.51 (SD 28.69). When all patient data were considered, the lowest score was 1.81 (SD 1.07) [39 $^{\text {th }}$ item (I enjoy feeding my child)], the highest score was 3.74 (SD 1.25) $\left[18^{\text {th }}\right.$ item (Medical professionals do not understand the care my child requires for feeding)].The floor effect for the total score was $0 \%$, the ceiling effect was $2.1 \%$, and both were acceptable values.

\section{Reliability}

Cronbach's alpha value of the scale was 0.821 . Corrected item-to-total correlations ranged from 0.729 to 0.911 . Removal of the substance did not cause any increase above Cronbach's alpha. For test-retest reliability coefficients calculated with ICC, the total score was 0.989, ranging from 0.941 to 0.995 (Table III).

\section{Validity}

A negative significant good level correlation was found between the dysphagia level by using endoscopic evaluation and the T- PFSDFIS total score as well as between total scores of IFS and T- PFSDFIS (r:-0.704, $\mathrm{p}=0.007, \mathrm{r}: 0.721, \mathrm{p}=0.001$, respectively). 
Table I. Caregivers' Demographics.

\begin{tabular}{|c|c|}
\hline & $\begin{array}{l}\text { Caregiver } \mathrm{n}=251 \\
\text { mean (SD), } \mathrm{n}(\%)\end{array}$ \\
\hline Age (years) & $34.24(10.55)$ \\
\hline \multicolumn{2}{|l|}{ Gender } \\
\hline Female & $249(99.2)$ \\
\hline Male & $2(0.8)$ \\
\hline \multicolumn{2}{|l|}{ Education } \\
\hline Illiterate & $1(0.4)$ \\
\hline Under 5-years & $29(11.6)$ \\
\hline 5 -years & $71(28.3)$ \\
\hline 8-years & $78(31.1)$ \\
\hline 11 years & $52(20.7)$ \\
\hline More than 11 years & $20(7.9)$ \\
\hline \multicolumn{2}{|l|}{ Marital status } \\
\hline Married & $238(94.8)$ \\
\hline Single & 0 \\
\hline Divorced & $13(5.2)$ \\
\hline $\begin{array}{l}\text { The numbers of people in the } \\
\text { house }\end{array}$ & $4.78(1.27)$ \\
\hline $\begin{array}{l}\text { The numbers of children in the } \\
\text { house }\end{array}$ & $2.15(1.18)$ \\
\hline \multicolumn{2}{|l|}{ Family income } \\
\hline High income level & $46(18.3)$ \\
\hline Moderate income level & $107(42.6)$ \\
\hline Low income level & $98(39.1)$ \\
\hline
\end{tabular}

Distribution of total score of T-PFSDFIS according to the dysphagia level by using FEES is shown in Table IV. In subgroup analysis; the lowest value was in normal swallowing and significantly different from all dysphagia levels. (with mild $\mathrm{p}=0.033$, with moderate $p=0.001$, with severe $p=0.001$, respectively). Similarly, the difference between mild and both moderate $(p=.002)$ and severe $(p=0.011)$ was significant. The difference between moderate and severe was not significant $(p=0.135)$.

\section{Discussion}

There is no specific scale in Turkish with documented validity and reliability that assesses how the families of children with $\mathrm{CP}$ with swallowing disorder are affected.
Table II. Children's Demographics and Disease Characteristics.

\begin{tabular}{|c|c|}
\hline & $\begin{array}{l}\text { Children } \mathrm{n}=251 \\
\text { mean }(\mathrm{SD}), \mathrm{n}(\%)\end{array}$ \\
\hline Age (months) & $68.11(20.26)$ \\
\hline \multicolumn{2}{|l|}{ Gender } \\
\hline Female & $79(31.5)$ \\
\hline Male & $172(68.5)$ \\
\hline BMI (\%) & $14.12(2.3)$ \\
\hline \multicolumn{2}{|l|}{ Additional problems } \\
\hline Mental retardation & $153(61)$ \\
\hline Seizure disorder & $133(53)$ \\
\hline Hearing disorder & $11(4.4)$ \\
\hline Vision disorder & $92(36.7)$ \\
\hline Speech disorder & $125(49.8)$ \\
\hline Gastrointestinal disorder & $174(69.3)$ \\
\hline Dental disorder & $152(60.6)$ \\
\hline \multicolumn{2}{|l|}{ Feeding type } \\
\hline Oral & $193(76.9)$ \\
\hline Enteral & $58(23.1)$ \\
\hline \multicolumn{2}{|l|}{ GMFM } \\
\hline I & 0 \\
\hline II & $27(11.8)$ \\
\hline III & $42(16.7)$ \\
\hline IV & $69(27.5)$ \\
\hline $\mathrm{V}$ & $113(45)$ \\
\hline \multicolumn{2}{|l|}{ CP type } \\
\hline Hemiparesis & $28(11.2)$ \\
\hline Diparesis & $41(16.3)$ \\
\hline Tetraparesis & $182(72.5)$ \\
\hline
\end{tabular}

SD: Standard deviation, BMI: body mass index, GMFM: Gross Motor Function Measure, CP: cerebral palsy

Therefore, this study aimed to translate and to conduct validity and reliability of PFSDFIS for caregivers of children with $\mathrm{CP}$.

For parents, the birth of a child is a great source of happiness, but it also brings new responsibilities and tasks in addition to those that existed before. $\mathrm{CP}$ is a disorder that causes motor, movement, balance and posture disorders from mild to severe. This condition begins in early childhood and persisting 
Table III. Antropometrics Characteristics,Pubertal Stages and Acantosis Nigricans Ratio of Study Population.

\begin{tabular}{|c|c|c|c|c|c|}
\hline \multirow[b]{2}{*}{ Items } & \multicolumn{3}{|c|}{ Test } & \multicolumn{2}{|c|}{ Test-retest } \\
\hline & Mean (SD) & Range & $\begin{array}{l}\text { Corrected } \\
\text { item-to-total } \\
\text { correlation }\end{array}$ & ICC & (\%95 CI) \\
\hline Item 1 & $3.11(1.57)$ & $1-5$ & 0.824 & 0.961 & $0.947-0.983$ \\
\hline Item 2 & $3.14(1.43)$ & $1-5$ & 0.833 & 0.972 & $0.955-0.980$ \\
\hline Item 3 & $3.18(1.66)$ & $1-5$ & 0.861 & 0.992 & 0.991-0.998 \\
\hline Item 4 & $3.25(1.58)$ & $1-5$ & 0.735 & 0.951 & $0.932-0.968$ \\
\hline Item 5 & $2.59(1.21)$ & $1-5$ & 0.737 & 0.990 & 0.985-0.994 \\
\hline Item 6 & $2.77(1.36)$ & $1-5$ & 0.810 & 0.972 & $0.964-0.981$ \\
\hline Item 7 & $2.59(1.59)$ & $1-5$ & 0.857 & 0.985 & $0.972-0.989$ \\
\hline Item 8 & $2.74(1.43)$ & $1-5$ & 0.833 & 0.995 & 0.989-0.997 \\
\hline Item 9 & $2.85(1.40)$ & $1-5$ & 0.825 & 0.948 & $0.923-0.961$ \\
\hline Item 10 & $2.77(1.45)$ & $1-5$ & 0.911 & 0.941 & $0.912-0.958$ \\
\hline Item 11 & $2.70(1.29)$ & $1-5$ & 0.764 & 0.955 & $0.943-0.971$ \\
\hline Item 12 & $2.70(1.40)$ & $1-5$ & 0.815 & 0.991 & $0.988-0.997$ \\
\hline Item 13 & $2.51(1.31)$ & $1-5$ & 0.894 & 0.967 & $0.952-0.972$ \\
\hline Item 14 & $2.37(1.44)$ & $1-5$ & 0.836 & 0.963 & $0.954-0.978$ \\
\hline Item 15 & $2.74(1.37)$ & $1-5$ & 0.835 & 0.991 & $0.987-0.994$ \\
\hline Item 16 & $3.29(1.29)$ & $1-5$ & 0.817 & 0.962 & $0.953-0.973$ \\
\hline Item 17 & $2.74(1.34)$ & $1-5$ & 0.834 & 0.989 & $0.956-0.993$ \\
\hline Item 18 & $3.74(1.25)$ & $1-5$ & 0.832 & 0.973 & $0.952-0.981$ \\
\hline Item 19 & $2.03(1.05)$ & $1-5$ & 0.729 & 0.976 & $0.961-0.979$ \\
\hline Item 20 & $2.44(1.31)$ & $1-5$ & 0.827 & 0.947 & $0.929-0.958$ \\
\hline Item 21 & $3.33(1.33)$ & $1-5$ & 0.829 & 0.991 & $0.987-0.995$ \\
\hline Item 22 & $2.55(1.31)$ & $1-5$ & 0.793 & 0.972 & $0.968-0.984$ \\
\hline Item 23 & $2.71(1.32)$ & $1-5$ & 0.732 & 0.979 & $0.962-0.988$ \\
\hline Item 24 & $2.74(1.22)$ & $1-5$ & 0.773 & 0.994 & 0.987-0.997 \\
\hline Item 25 & 2.59 (1.39) & $1-5$ & 0.755 & 0.954 & $0.927-0.960$ \\
\hline Item 26 & $2.70(1.38)$ & $1-5$ & 0.817 & 0.992 & $0.982-0.996$ \\
\hline Item 27 & $2.77(1.36)$ & $1-5$ & 0.781 & 0.968 & $0.957-0.975$ \\
\hline Item 28 & $2.85(1.43)$ & $1-5$ & 0.787 & 0.992 & $0.983-0.996$ \\
\hline Item 29 & $2.59(1.22)$ & $1-5$ & 0.838 & 0.987 & 0.981-0.992 \\
\hline Item 30 & $2.62(1.49)$ & $1-5$ & 0.849 & 0.981 & $0.969-0.991$ \\
\hline Item 31 & $3.07(1.54)$ & $1-5$ & 0.747 & 0.990 & $0.97-0.996$ \\
\hline Item 32 & $3.11(1.33)$ & $1-5$ & 0.744 & 0.983 & $0.978-0.989$ \\
\hline Item 33 & $1.85(1.02)$ & $1-5$ & 0.759 & 0.968 & $0.959-0.983$ \\
\hline Item 34 & $2.66(1.08)$ & $1-5$ & 0.834 & 0.972 & $0.961-0.979$ \\
\hline Item 35 & $2.18(1.27)$ & $1-5$ & 0.787 & 0.992 & $0.988-0.994$ \\
\hline Item 36 & $3.62(1.18)$ & $1-5$ & 0.802 & 0.959 & $0.941-0.967$ \\
\hline
\end{tabular}


Table III. Continue.

\begin{tabular}{lccccc}
\hline & \multicolumn{3}{c}{ Test } & \multicolumn{2}{c}{ Test-retest } \\
\cline { 2 - 6 } Items & Mean (SD) & Range & $\begin{array}{c}\text { Corrected } \\
\text { item-to-total } \\
\text { correlation }\end{array}$ & ICC & $(\% 95$ CI $)$ \\
\hline Item 37 & $3.40(1.00)$ & $1-5$ & 0.761 & 0.988 & $0.981-0.994$ \\
Item 38 & $2.48(1.12)$ & $1-5$ & 0.732 & 0.976 & $0.968-0.986$ \\
Item 39 & $1.81(1.07)$ & $1-5$ & 0.739 & 0.985 & $0.979-0.991$ \\
Item 40 & $2.55(1.33)$ & $1-5$ & 0.738 & 0.994 & $0.991-0.997$ \\
Item 41 & $2.88(1.42)$ & $1-5$ & 0.746 & 0.944 & $0.918-0.952$ \\
Item 42 & $2.85(1.51)$ & $1-5$ & 0.774 & 0.982 & $0.978-0.988$ \\
Item 43 & $2.44(1.31)$ & $1-5$ & 0.768 & 0.968 & $0.955-0.978$ \\
Item 44 & $2.85(1.16)$ & $1-5$ & 0.748 & 0.993 & $0.990-0.996$ \\
Total score & $122.51(28.69)$ & $44-220$ & 0.821 & 0.989 & $0.941-0.995$ \\
\hline
\end{tabular}

T-PFSDFIS: Turkish version- Pediatric Feeding and Swallowing Disorders Family Impact Scale, SD: Standard deviation, ICC: intra-class correlation coefficient.

Table IV. Distribution of Total Score of T-PFSDFIS According to the Dysphagia Level by Using FEES.

\begin{tabular}{lcccc}
\hline & Normal & Mild Level & Moderate level & Severe level \\
$\mathrm{n}=29$ & $\mathrm{n}=72$ & $\mathrm{n}=79$ & $\mathrm{n}=71$ \\
& Mean (SD) & Mean (SD) & Mean (SD) & Mean (SD) \\
\hline T-PFSDFIS (total score) & $162.66(11.37)$ & $147.75(5.39)$ & $105.10(8.52)$ & $99.57(25.15)$ \\
\hline
\end{tabular}

SD: standard deviation, T-PFSDFIS: Turkish version-Pediatric Feeding and Swallowing Disorders Family Impact Scale Normal; FEES score:1, Mild; FEES score 2-3, Moderate; FEES score:4-5, severe; FEES score:6.

throughout the lifespan and partially or fully dependent children need caregivers. Although many caregivers cope with this new condition in the presence of $\mathrm{CP}$, the lack of complete recovery of disorders leads to physical and psychological impact on caregivers. ${ }^{18,19}$

Therefore, many scales have been developed where general physical and psychological health of children and their families are discussed, but in some of these scales ${ }^{20}$, family relationships at different levels, meal times and financial issues are not addressed or some of them included studies that were more specific (unemployed, single parents, etc.), or have limited evaluations to reduce the number of questions. ${ }^{15}$ However, in order to identify how the caregiver is affected by a child with disability, rather than focusing on simply measuring satisfaction the assessment of wider aspects of event impact is needed. For this reason, we have chosen the PFSDFIS scale to better understand the problems of caregivers of children with CP.
The Pediatric Feeding and Swallowing Disorders Family Impact Scale (PFSDFIS) was developed to help clinicians identify the impact feeding and swallowing problems have on the families of children with chronic illness by Redle. ${ }^{12}$ Cronbach's alpha indicated good internal consistency (cronbach $\alpha=0.92$ ). Scores for item distribution indicated a large number of items with floor and ceiling effects which may impact the scale's sensitivity to measuring change. Validation on this scale was made by comparing with caregivers of healthy children and reported to be effective as well as it was reported that the total score should be evaluated instead of the subgroups because of the multiplicity of questions.

But an objective method such as endoscopic evaluation has not been used. As a result, the scale which we used in the families of children with CP demonstrated acceptable internal consistensy level (cronbach $\alpha=0.821$ ). The total-score-level test-retest reliability was very good (ICC: 0.989). Also, the floor effect 
for the total score was $0 \%$, the ceiling effect was $2.1 \%$, and both were acceptable. In our study, validation was assessed with both FEES evaluating dysphagia objectively and another impact scale. According to this, a negative significant good level correlation was found between the dysphagia level by using endoscopic evaluation and the T- PFSDFIS total score as well as between total scores of IFS and T- PFSDFIS. With subgroup analysis; it was found that increased degree of dysphagia is associated with the lower scores, in other words, as the swallowing disorder worsened; it was also possible to show that the impact was increased.

This study has another contribution to the literature. This scale is effective in reflecting the influence of caregivers on the severity of dysphagia measured objectively (endoscopic method).

The limitations of our study are that it was carried out in a single center, and we did not include caregivers of healthy children. We believe that studies involving multicentre and healthy children in the future will provide a better understanding of our results.

Consequently, T-PFSDFIS is a valid and reliable scale to show comprehensive impact of caring for children with $\mathrm{CP}$ and swallowing disorder in Turkey.

\section{REFERENCES}

1. Stavsky M, Mor O, Mastrolia SA, Greenbaum S, Than NG, Erez O. Cerebral palsy-trends in epidemiology and recent development in prenatal mechanisms of disease, treatment, and prevention. Front Pediatr 2017; 5: 21.

2. Rosenbaum P, Paneth N, Leviton A, et al. A report: the definition and classification of cerebral palsy. Dev Med Child Neurol Suppl 2007; 109: 8-14.

3. Strauss D, Brooks J, Rosenbloom L, Shavelle R. Life expectancy in cerebral palsy: an update. Dev Med Child Neurol 2008; 50: 487-493.

4. Cowpe Jebson E, Hanson B, Smith $\mathrm{CH}$. What do parents of children with dysphagia think about their MDT? A qualitative study. BMJ Open 2014; 4: e005934.

5. Chambers HG, Chambers JA. Effects of caregiving on the families of children and adults with disabilities. Phys Med Rehabil Clin N Am 2015; 26: 1-19.
6. Lagos-Guimarães HNC, Teive HAG, Celli A, et al. Aspiration pneumonia in children with cerebral palsy after videofluoroscopic swallowing study. Int Arch Otorhinolaryngol 2016; 20: 132-137.

7. Ballantyne M, Leslie P, Redle E. Paediatric dysphagia, parenting stress and feeding tubes. J Oral Health Dent 2017; 1(S1): A0012.

8. Serdaroğlu A, Cansu A, Ozkan S, Tezcan S. Prevalance of cerebral palsy in Turkish children between the ages of 2 and 16 years. Dev Med Child Neurol 2006; 48: 413-416.

9. Erkin G, Delialioglu SU, Ozel S, Culha C, Sirzai H. Risk factors and clinical profiles in Turkish children with cerebral palsy: analysis of 625 cases. Int J Rehabil Res 2008; 31: 89-91.

10. Aydin K; Turkish Cerebral Palsy Study Group. A multicenter cross-sectional study to evaluate the clinical characteristics and nutritional status of children with cerebral palsy. Clin Nutr ESPEN 2018; 26: 27-34.

11. Umay E, Unlu E, Cakci A. Psychological status and quality of life of caregivers of children with dysphagic cerebral palsy. Research 2014; 1: 768 .

12. Redle EE. The Pediatric Feeding and Swallowing Disorders Family Impact Scale: Scale Development and Initial Psychometric Properties. Unpublished Dissertation, University of Cincinnati, Cincinnati, 2007.

13. Beaton DE, Bombardier C, Guillemin F, Ferraz MB. Guidelines for the process of cross-cultural adaptation of self-report measures. Spine (Phila Pa 1976) 2000; 25: 3186-3191.

14. Warnecke T1, Teismann I, Oelenberg S, et al. Towards a basic endoscopic evaluation of swallowing in acute stroke-identification of salient findings by the inexperienced examiner. BMC Med Educ 2009; 9: 13.

15. Stein RE, Riessman CK. The development of an impact-on-family scale: preliminary findings. Med Care 1980; 18: 465-472.

16. Bowling A. Quality of life: measures and meanings in social care research. Methods Review 16. NIHR School for Social Care Research, London 2014: 13-21.

17. Bland JM, Altman DG. Statistical methods for assessing agreement between two methods of clinical measurement. Lancet 1986; 8476: 307-310.

18. Pousada M, Guillamón N, Hernández-Encuentra E, et al. Impact of caring for a child with cerebral palsy on the quality of life of parents: A systematic review of the literature. J Dev Phys Disabil 2013; 25: 545-577.

19. Park EY, Nam SJ. Time burden of caring and depression among parents of individuals with cerebral palsy. Disabil Rehabil 2019; 41: 1508-1513.

20. Varni JW, Sherman SA, Burwinkle TM, Dickinson PE, Dixon P. The PedsQL family impact module: preliminary reliability and validity. Health Qual Life Outcomes 2004; 2: 55. 